Research Paper

\title{
A Nomogram Predicting Extrahepatic Metastases for Patients with Adjuvant Transarterial Chemoemboli- zation after Hepatectomy
}

\author{
Shipeng Chen ${ }^{1}{ }^{*}$, Yuzhen $\mathrm{Gao}^{3 *}$, Zheng Li14 Jian'an Jia1, Meng Fang1, Mengmeng Wang1, Huijuan Feng1, \\ Qinjunjie Chen ${ }^{4}$, Wenqian Guan ${ }^{1,2}$, Ziyi Wang5 ${ }^{5}$, Chunfang Gao ${ }^{\bowtie}$ \\ 1. Department of Laboratory Medicine, Third Affiliated Hospital of Second Military Medical University, Shanghai, China \\ 2. Clinical Medical College, Fujian Medical University, Fuzhou, China \\ 3. Department of Molecular Diagnosis, Clinical Medical College, Yangzhou University, Yangzhou, China \\ 4. Department of Hepatic Surgery, Third Affiliated Hospital of Second Military Medical University, Shanghai, China \\ 5. Shanghai Institute of Technology, Shanghai, China \\ *These authors have contributed equally to this work. \\ $\square$ Corresponding author: Chunfang Gao, M.D. \& Ph.D, Department of Laboratory Medicine, Third Affiliated Hospital of Second Military Medical University, \\ 225 Changhai Road, Shanghai, 200438, China. E-mail: gaocf1115@163.com. \\ (1) Ivyspring International Publisher. This is an open access article distributed under the terms of the Creative Commons Attribution (CC BY-NC) license \\ (https:// creativecommons.org/licenses/by-nc/4.0/). See http://ivyspring.com/terms for full terms and conditions.
}

Received: 2018.03.05; Accepted: 2018.08.15; Published: 2018.10.20

\begin{abstract}
Background: Prognosis remains poor for hepatocellular carcinoma $(\mathrm{HCC})$ patients with extrahepatic metastases (EHMs). This study aimed to develop a nomogram to predict EHMs in HCC patients who underwent adjuvant transarterial chemoembolization (TACE) following hepatectomy.

Methods: Data of 578 HCC patients who underwent TACE after hepatectomy at the Eastern Hepatobiliary Surgery Hospital was retrospectively reviewed. Cox regression analyses was used to select variables to construct the nomogram. Predictive accuracy and discriminative ability of the model were performed using concordance index ( $\mathrm{C}$-index), calibration curve and the area under time-dependent receiver operating characteristic (ROC) curve.

Results: Postoperative EHMs were detected in 89 and 31 patients in the training cohort $(n=453)$ and validation cohort $(n=125)$, respectively. Multivariate analysis showed that tumor size $(\mathrm{HR}, 1.099 ; 95 \% \mathrm{Cl}$, 1.049-1.152), coarse beam type of tumor histopathological structure (HR, 2.382; 95\% Cl, 1.030-5.512), presence of satellite nodules (HR, 1.936; 95\% Cl, 1.156-3.244) and alpha-fetoprotein (AFP) (HR, 1.399; $95 \% \mathrm{Cl}, 1.098-1.783$ ) were independent risk factors for EHMs (all $\mathrm{p}<0.05$ ). The nomogram incorporated these factors achieved good agreement between prediction and actual observation with a concordance index (C-index) of 0.73 ( $95 \% \mathrm{Cl}, 0.68$ to 0.78$)$ and $0.71(95 \% \mathrm{Cl}, 0.63$ to 0.79$)$ in the training cohort and validation cohort, respectively. In addition, patients who had a nomogram score $>17$ were considered to have higher risk for EHMs compared with those scored $\leq 12$. Furthermore, the time-dependent area under the ROC curve indicated comparative stability and adequate discriminative ability of the model.

Conclusions: This novel nomogram can identify those with high risk of EHMs after adjuvant TACE following hepatectomy. The validation cohort showed a good performance, suggesting it could benefit surgeons on decision-making.
\end{abstract}

Key words: Hepatocellular carcinoma, Adjuvant transarterial chemoembolization, Extrahepatic metastases, Nomogram

\section{Introduction}

Hepatocellular carcinoma (HCC) is the sixth most prevalent malignancy and the third most frequent cause of cancer-related death worldwide [1].
Although hepatectomy and liver transplantation remain the main treatments for resectable tumors, the prognosis for HCC is still poor. HCC patients could 
only achieve a 5-year survival rate nearly 33\% - 50\% through resection, while the 5-year recurrence rate after surgical resection is more than $75 \%$ [1]. In many randomized trials, it was reported that patients who received adjuvant transarterial chemoembolization (TACE) after hepatectomy had significantly higher survival rate [2, 3]. Nonetheless, extrahepatic metastases (EHMs) were observed more frequently because of the prolonged survival, with an incidence of $13.5 \%$ to $42 \%$ in HCC patients [4].

Therapeutic options for EHMs are comparatively limited currently, especially for diffuse EHMs [5]. HCC can metastasize to many parts of the body by way of blood circulation and direct invasion of lymphatic spread. Patients with EHMs from primary HCC are considered to be in the terminal stage of cancer; accordingly, the prognosis for them continues to be poor. Recently, the management of EHMs in the lung, bone and brain has been improved, leading to better outcomes than before [6-8]. Moreover, patients can benefit from adjuvant therapies, including surgical, adoptive immunotherapy, antiviral therapy and radiotherapy [9-12]. For example, it reported that external radiotherapy can slow tumor progression and prolong survival for patients with EHMs [13-15]. Besides, Llovet and Kudo $\mathrm{M}$ et al reported that sorafenib and lenvatinib could extend survival in the treatment of advanced HCC, respectively $[16,17]$. In addition, oxaliplatin can benefit patients with metastatic liver cancer [18]. Furthermore, Qiu et al reported that the thymalfasin can significantly prolong remission in patients who received adjuvant TACE after tumor resection [19]. Most importantly, accumulated evidence suggests that Huaier may be associated with inhibition of cell proliferation, anti-metastasis, interference with tumor angiogenesis and tumor-specific immunomodulatory effect [20]. It may reduce metastasis and improve survival by strengthening immune function. Consequently, it makes sense to identify patients who received TACE after hepatectomy are at high risk for EHMs. Such EHMs profile would prove essential for surgeons planning potential adjuvant treatments, as noted above, and other novel therapies at an earlier stage. Furthermore, identifying patients at high risk for EHMs could concurrently support the early detection of lesions, thus maximizing the preventive benefits of treatment through early diagnosis and treatment as well as preventing unnecessary liver transplantation in these patients.

Currently, the realistic methods for reducing EHMs after resection include early detection and aggressive management of it. Although significant progress has been made in imaging technology, many deficiencies limit its use for EHMs from HCC, not to mention those patients with no early signs. EHMs from HCC are hard to predict based on routine clinical assay, but some specific and predictive method could provide early detection or even prevention of it. In this regard, the nomogram is a tool that generates the probability of an individual clinical event by integrating diverse prognostic and determinative variables [21]. Therefore, in the present research, we constructed a nomogram from a training cohort of 453 patients to predict the probability of EHMs in HCC patients who received TACE after hepatectomy. The nomogram was further validated in another independent cohort of 125 patients.

\section{Patients and Methods}

\section{Patient inclusion and exclusion}

The flowchart of enrolled patients underwent partial hepatectomy for HCC and adjuvant TACE in the Eastern Hepatobiliary Surgery Hospital (Figure 1). From March 2009 to December 2012, a cohort of 453 patients diagnosed with HCC by histopathology, according to EASL criteria [22], underwent partial hepatectomy for HCC and adjuvant TACE at Department of Hepatic Surgery of Eastern Hepatobiliary Surgery Hospital were enrolled. Inclusion criteria included the following: no history of preoperative anticancer therapy; no EHMs at the time of diagnosis; no history of other malignancies; no macrovascular invasion; R0 resection; and pathological diagnosis of HCC in all resected tumors. In addition, we checked all the patients again at our hospital 4 weeks after partial hepatectomy. Then TACE therapy was appropriately recommended according to the liver function of the patients if no recurrence was found. Patients diagnosed with recurrence at that time were excluded, and in order to avoid counting deaths due to postoperative complications, patients died within 30 days post-hepatectomy were also excluded. Besides, the study was also conducted on another separate cohort of 125 patients. These patients, who met the same inclusion and exclusion criteria mentioned above, were considered as the validation cohort.

\section{Data collection}

All patients enrolled in this study were evaluated with a baseline history and physical examination at the discretion of the treating surgeons. All laboratory tests, such as alpha-fetoprotein (AFP) as well as liver function parameters, were calculated before the hepatectomy. Besides, appropriate pre-surgical imaging, such as dynamic contrast enhanced computed tomography (CT) and/or magnetic resonance imaging (MRI) of liver, 
abdominal ultrasound, chest CT were performed before hepatectomy. In addition, bone scan were performed when hepatocellular carcinoma patients with signs and symptoms of bone metastasis including: bone pain, fractures, spinal cord compression and hypercalcemia et al.

Histopathological study of the resected specimens, including tumor diameter and number, degree of cirrhosis, microvascular invasion and the status of tumor encapsulation, was carried out independently by two experienced pathologists. In the event of disagreement, deference was given to the senior pathologist, but only when consensus was reached would histopathology be decided.

\section{Follow up}

Every enrolled patient was followed up every 3 months during the first 2 years after hepatectomy and then every 3 - 6 months thereafter until death or dropout from our program. Overall survival was defined as the interval between hepatectomy and death, or the last date of follow-up program. EHMs were diagnosed based on a patient's CT/MRI scan, and time to development of EHMs was calculated as the time from hepatectomy to the date when EHMs were first confirmed. The median (interquartile range) postoperative follow-up time was 40.47 (24.03-48.93) months in all patients. The diagnostic criteria for EHMs were as follows: (1) raised AFP that had declined to normal range after hepatectomy, (2) evidence of new extrahepatic lesions not found previously, (3) histopathological study of extrahepatic lesions in patients who underwent re-resection for recurrence of HCC. Patients with intrahepatic recurrence prior to EHMs, or concurrent with EHMs, were excluded in this study. Patients diagnosed with EHMs and other malignancies were also excluded. Active treatment plans were implemented according to liver function status and tumor number when recurrence was confirmed.

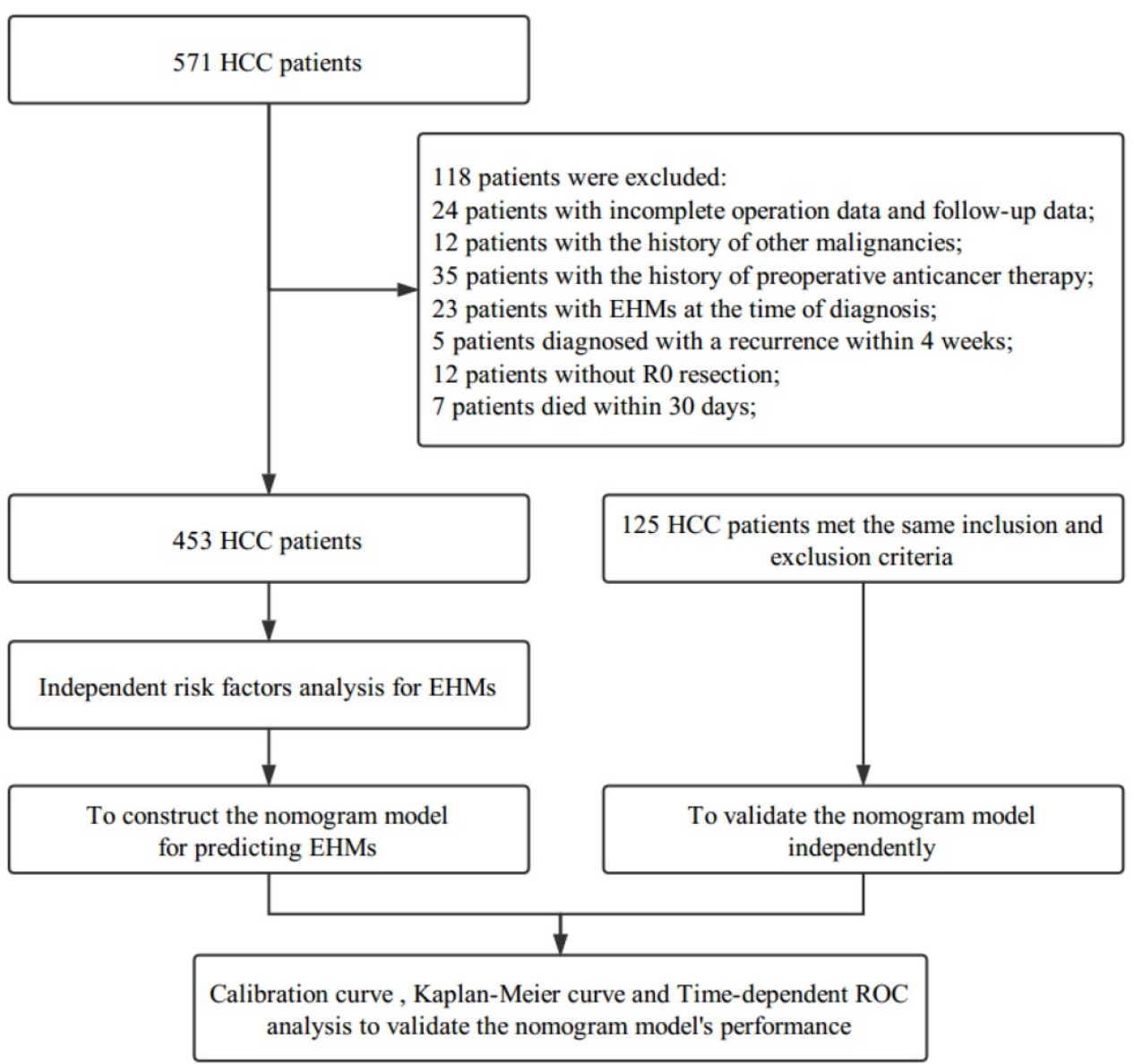

Figure 1. The flowchart of the enrolled patients 
Table 1. Baseline characteristics of HCC patients in the training and validation cohorts

\begin{tabular}{|c|c|c|c|}
\hline Variables & Training Cohort $(n=453)$ & Validation Cohort $(n=125)$ & P-value \\
\hline Age (years) & $50.81 \pm 9.77$ & $49.37 \pm 11.39$ & 0.160 \\
\hline Gender (\%) & & & 0.255 \\
\hline Male & $384.00(84.77)$ & $111.00(88.80)$ & \\
\hline Female & $69.00(15.23)$ & $14.00(11.20)$ & \\
\hline Tumor size (cm) & $5.20(3.60-8.00)$ & $4.90(3.40-8.15)$ & 0.901 \\
\hline State of recurrence $(\%)$ & & & 0.146 \\
\hline Presence & $206.00(45.47)$ & $66.00(52.80)$ & \\
\hline Absence & $247.00(54.53)$ & $59.00(47.20)$ & \\
\hline Extrahepatic metastases (\%) & & & 0.213 \\
\hline Lung & $47.00(10.38)$ & $14.00(11.20)$ & \\
\hline Bone & $8.00(1.77)$ & $3.00(2.40)$ & \\
\hline Lung \& Bone & $15.00(3.31)$ & $2.00(1.60)$ & \\
\hline Others & $19.00(4.19)$ & $12.00(9.60)$ & \\
\hline Number of tumor $(\%)$ & & & 0.675 \\
\hline Single & $362.00(79.91)$ & $102.00(81.60)$ & \\
\hline Multiple & $91.00(20.09)$ & $23.00(18.40)$ & \\
\hline Tumor encapsulation (\%) & & & 0.120 \\
\hline Complete & $224.00(49.45)$ & $52.00(41.60)$ & \\
\hline Incomplete & $229.00(50.55)$ & $73.00(58.40)$ & \\
\hline Liver cirrhosis (\%) & & & 0.793 \\
\hline Presence & $256.00(56.51)$ & $69.00(55.20)$ & \\
\hline Absence & $197.00(43.49)$ & $56.00(44.80)$ & \\
\hline Microvascular invasion (\%) & & & 0.434 \\
\hline Presence & $171.00(37.75)$ & $52.00(41.60)$ & \\
\hline Absence & $282.00(62.25)$ & $73.00(58.40)$ & \\
\hline Histopathological structure of tumor $(\%)$ & & & 0.995 \\
\hline Coarse beam type & $377.00(83.22)$ & $104.00(83.20)$ & \\
\hline Fine beam type & $76.00(16.78)$ & $21.00(16.80)$ & \\
\hline Tumor differentiation (\%) & & & 0.950 \\
\hline Lower & $2.00(0.44)$ & $1.00(0.80)$ & \\
\hline Low & $71.00(15.67)$ & $19.00(15.20)$ & \\
\hline Moderate & $359.00(79.25)$ & $100.00(80.00)$ & \\
\hline High & $21.00(4.64)$ & $5.00(4.00)$ & \\
\hline Satellite nodules $(\%)$ & & & 0.416 \\
\hline Presence & $53.00(11.70)$ & $18.00(14.40)$ & \\
\hline Absence & $400.00(88.30)$ & $107.00(85.60)$ & \\
\hline HBsAg (\%) & & & 0.955 \\
\hline Positive & 336.00 (74.17) & $92.00(73.60)$ & \\
\hline Negative & $41.00(9.05)$ & $11.00(8.80)$ & \\
\hline HBV DNA (log IU/mL) & $3.24(3.00-5.03)$ & $3.33(3.00-5.22)$ & 0.653 \\
\hline $\mathrm{TP}(\mathrm{g} / \mathrm{L})$ & $70.20(65.30-74.95)$ & $71.30(65.70-75.75)$ & 0.258 \\
\hline $\operatorname{ALB}(\mathrm{g} / \mathrm{L})$ & $40.90(37.50-43.30)$ & $41.00(37.00-44.15)$ & 0.557 \\
\hline TBIL (umol/L) & $13.90(10.65-18.45)$ & $14.10(10.75-18.30)$ & 0.824 \\
\hline GGT (U/L) & $63.00(36.25-116.50)$ & $63.00(36.50-102.50)$ & 0.966 \\
\hline AFU (U/L) & $25.00(21.00-32.50)$ & $27.00(21.00-33.00)$ & 0.407 \\
\hline $\operatorname{ALP}(\mathrm{U} / \mathrm{L})$ & $83.00(69.00-106.00)$ & $83.00(67.00-106.50)$ & 0.450 \\
\hline $\operatorname{ALT}(\mathrm{U} / \mathrm{L})$ & $40.80(27.70-90.50)$ & $44.40(23.55-75.50)$ & 0.555 \\
\hline AST (U/L) & $40.00(28.00-86.95)$ & $39.50(27.35-74.10)$ & 0.650 \\
\hline PT (s) & $12.20(11.50-12.80)$ & $12.10(11.40-12.80)$ & 0.622 \\
\hline $\operatorname{PLT}\left(10^{9} / \mathrm{L}\right)$ & $158.00(118.00-205.00)$ & $157.00(127.50-208.00)$ & 0.541 \\
\hline AFP (log ug/L) & $2.32(1.10-3.08)$ & $2.23(1.34-3.05)$ & 0.892 \\
\hline CEA (ug/L) & $2.20(1.50-3.48)$ & $2.10(1.40-3.25)$ & 0.389 \\
\hline CA19-9 (U/mL) & $18.10(10.57-30.45)$ & $16.90(10.35-31.10)$ & 0.976 \\
\hline
\end{tabular}

Categorical variables presented as number (percentage) and continuous data presented as means \pm standard deviations or median (interquartile range). Categorical variables were compared using the Chi-square test or Fisher's exact test. Continuous variables were compared using the independent t-test or Mann-Whitney U test. HBsAg: hepatitis B surface antigen; TP: total protein; ALB, albumin; TBIL: total bilirubin; GGT: $\gamma$-glutamyltransferase; AFU: a-L-fucosidase; ALP: alkaline phosphates; ALT: alanine aminotransferase; AST: aspartate aminotransferase; PT: prothrombin time; PLT: platelet count; AFP: alpha-fetoprotein; CEA: carcino-embryonic antigen; CA19-9: carbohydrate antigen 19-9.

\section{Statistical analysis}

Independent samples $t$-test or Mann-Whitney $\mathrm{U}$ test was used to calculate the differences between different sets in continuous variables. Categorical variables were compared using the Chi-square test or Fisher's exact test. Based on the results of the multivariate Cox regression analysis, we used $\mathrm{R}$ program packages, including "rcspline", "rms", "timeROC", "survivalROC", and "party", to construct and validate the nomogram model. The "rcspline" (restricted cubic splines) package was used to analyze the nonlinear effect among risk factors on EHMs. "rms" was used to draw the nomogram and validate 
this model which predicted the probability of EHMs. The predictive performance of the model in continuous time were expressed with the area under time-dependent ROC curve (AUC) by the "timeROC" package. Decision tree analysis in the "party" package was used to better stratify HCC patients into three groups with different EHMs probabilities both in the training and validation cohorts. Furthermore, the EHMs rates were compared using the Kaplan-Meier method and log-rank test among the high, moderate and low risk groups. A two-sided $p$ value of less than 0.05 was considered statistically significant. All statistical analyses and graphics in this study were performed in SPSS 23.0 (SPSS, Chicago, IL, USA), GraphPad Prism 7.0 (GraphPad Software, La Jolla, CA, USA) and R-3.3.0 for Windows (https:// www.rproject.org/).

\section{Results}

\section{Baseline characteristics of patients}

Baseline characteristics of the patients are shown in Table 1 . In the training cohort $(n=453)$, the mean age of patients was 50.81 years among whom 384 $(84.77 \%)$ was male. Most people $336(74.17 \%)$ were positive for hepatitis B virus surface antigen, and 256 $(56.51 \%)$ of these patients presented with liver cirrhosis. In terms of tumor factors, 362 (79.91\%) of the patients had single tumor, and the median diameter of the tumors was 5.20 (interquartile range: $3.60-8.00$ ) $\mathrm{cm}$. In addition, encapsulation incomplete and microvascular invasion were documented in 229 $(50.55 \%)$ and $171(37.75 \%)$ patients, respectively. As

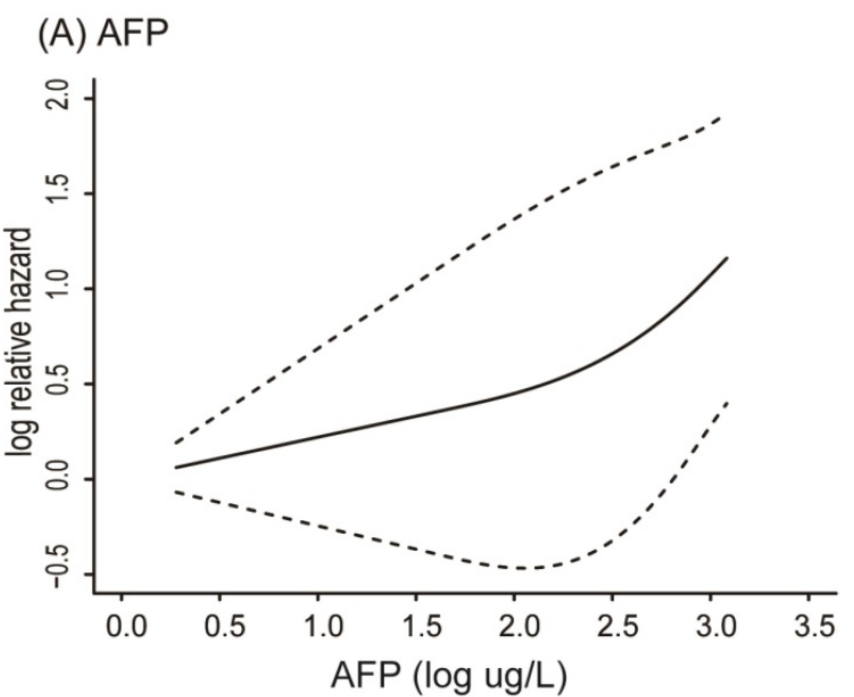

for postoperative prognosis, the rate of tumor recurrence was $206(45.47 \%)$ in the training cohort. All clinical and histopathological factors of the training cohort $(n=453)$ and validation cohort $(n=125)$ are summarized in Table 1. No significant differences in baseline characteristics were found between the training and validation cohorts.

\section{Independent factors associated with EHMs in the training cohort}

Table 2 shows the results of the univariate and multivariate analyses with the Cox proportional hazard regression model. It showed that tumor size (HR, 1.099; 95\% CI, 1.049-1.152), coarse beam type of tumor histopathological structure (HR, 2.382; 95\% CI, 1.030-5.512), presence of satellite nodules (HR, 1.936; 95\% CI, 1.156-3.244), and log AFP (HR, 1.399; 95\% CI, 1.098-1.783) were four independent variables associated with EHMs (all $\mathrm{p}<0.05)$. Histopathological structure of tumor was mainly divided into two categories: coarse beam type and fine beam type. In addition, before finally selecting variables for input, the influence of AFP and tumor size were performed by using restricted cubic splines. Both AFP and tumor size had nonlinear influence on the hazard ratio of EHMs. Using the R package "rcspline", we noted that the effect of AFP on the HR was at first linear below a threshold of almost $2 \log (\mathrm{AFP}) \mathrm{ug} / \mathrm{L}$, but after that it then increased dramatically (Figure 2A). Similarly, the effect of tumor size on the HR was linear, just below almost $6 \mathrm{~cm}$, and then grew gradually (Figure 2B).

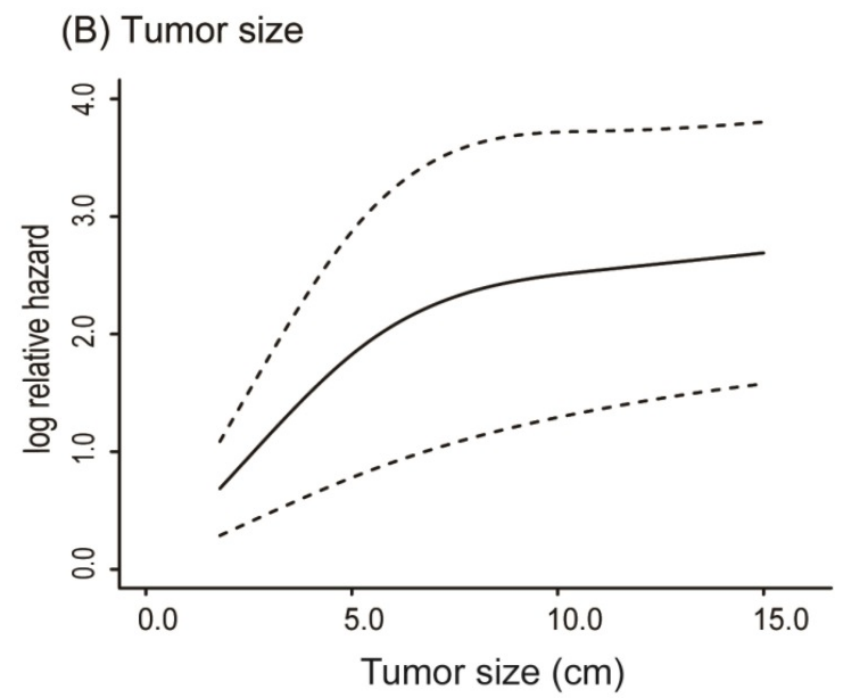

Figure 2. Nonlinear influence of AFP $(A)$ and tumor size $(B)$ on the hazard ratio of EHMs 
Table 2. Cox proportional hazards regression model showing the factors associated with extrahepatic metastasis of $\mathrm{HCC}$ in the training cohort $(\mathrm{N}=453)$

\begin{tabular}{|c|c|c|c|c|c|c|}
\hline \multirow[t]{2}{*}{ Variables } & \multicolumn{3}{|c|}{ Univariate } & \multicolumn{3}{|c|}{ Multivariate } \\
\hline & HR & $95 \% \mathrm{CI}$ & P-value & HR & $95 \% \mathrm{CI}$ & P-value \\
\hline Age (years) & 0.998 & 0.977-1.019 & 0.845 & & & \\
\hline Gender (male) & 0.621 & $0.374-1.030$ & 0.065 & & & \\
\hline HBsAg (positive) & 1.305 & $0.599-2.843$ & 0.503 & & & \\
\hline Tumor size $(\mathrm{cm})$ & 1.123 & 1.073-1.175 & $<0.001$ & 1.099 & $1.049-1.152$ & $<0.001$ \\
\hline Microvascular invasion (presence) & 1.854 & $1.223-2.810$ & 0.004 & & & \\
\hline Tumor encapsulation (incomplete) & 1.991 & $1.290-3.072$ & 0.002 & & & \\
\hline Histopathological structure of tumor (coarse beam type) & 3.212 & $1.402-7.361$ & 0.006 & 2.382 & $1.030-5.512$ & 0.043 \\
\hline Tumor differentiation (low) & 1.714 & $1.073-2.739$ & 0.024 & & & \\
\hline Satellite nodules (presence) & 2.505 & $1.508-4.163$ & $<0.001$ & 1.936 & $1.156-3.244$ & 0.012 \\
\hline Number of tumors (multiple) & 1.611 & $1.009-2.575$ & 0.046 & & & \\
\hline Liver cirrhosis (presence) & 1.030 & $0.676-1.571$ & 0.890 & & & \\
\hline HBV DNA $(\log I U / m L)$ & 0.979 & $0.813-1.179$ & 0.824 & & & \\
\hline $\mathrm{TP}(\mathrm{g} / \mathrm{L})$ & 0.991 & $0.964-1.017$ & 0.483 & & & \\
\hline TBIL (umol/L) & 1.009 & $0.983-1.036$ & 0.489 & & & \\
\hline $\operatorname{ALB}(\mathrm{g} / \mathrm{L})$ & 0.957 & 0.919-0.997 & 0.034 & & & \\
\hline $\operatorname{ALT}(\mathrm{U} / \mathrm{L})$ & 1.000 & $1.000-1.001$ & 0.415 & & & \\
\hline AST (U/L) & 1.000 & $1.000-1.001$ & 0.200 & & & \\
\hline GGT (U/L) & 1.001 & $1.000-1.002$ & 0.164 & & & \\
\hline AFU (U/L) & 1.001 & $0.980-1.022$ & 0.919 & & & \\
\hline $\operatorname{ALP}(\mathrm{U} / \mathrm{L})$ & 1.003 & $1.001-1.005$ & 0.003 & & & \\
\hline $\mathrm{PT}(\mathrm{s})$ & 0.985 & $0.828-1.171$ & 0.860 & & & \\
\hline $\operatorname{AFP}(\log u g / L)$ & 1.522 & $1.198-1.934$ & 0.001 & 1.399 & $1.098-1.783$ & 0.007 \\
\hline CEA (ug/L) & 0.994 & $0.956-1.032$ & 0.742 & & & \\
\hline CA19-9 (U/mL) & 1.001 & $0.991-1.011$ & 0.812 & & & \\
\hline PLT $\left(10^{9} / \mathrm{L}\right)$ & 1.002 & 0.998-1.005 & 0.338 & & & \\
\hline
\end{tabular}

CI: confidence interval; HR: hazard ratio; HBsAg: Hepatitis B surface antigen; TP: total protein; TBIL: total bilirubin; ALB: albumin; ALT: alanine aminotransferase; AST: aspartate aminotransferase; GGT: $y$-glutamyltransferase; AFU: $\alpha$-L-fucosidase; ALP: alkaline phosphates; PT: prothrombin time; AFP: a-fetoprotein; CEA: carcino-embryonic antigen; CA19-9: carbohydrate antigen 19-9; PLT: platelets.

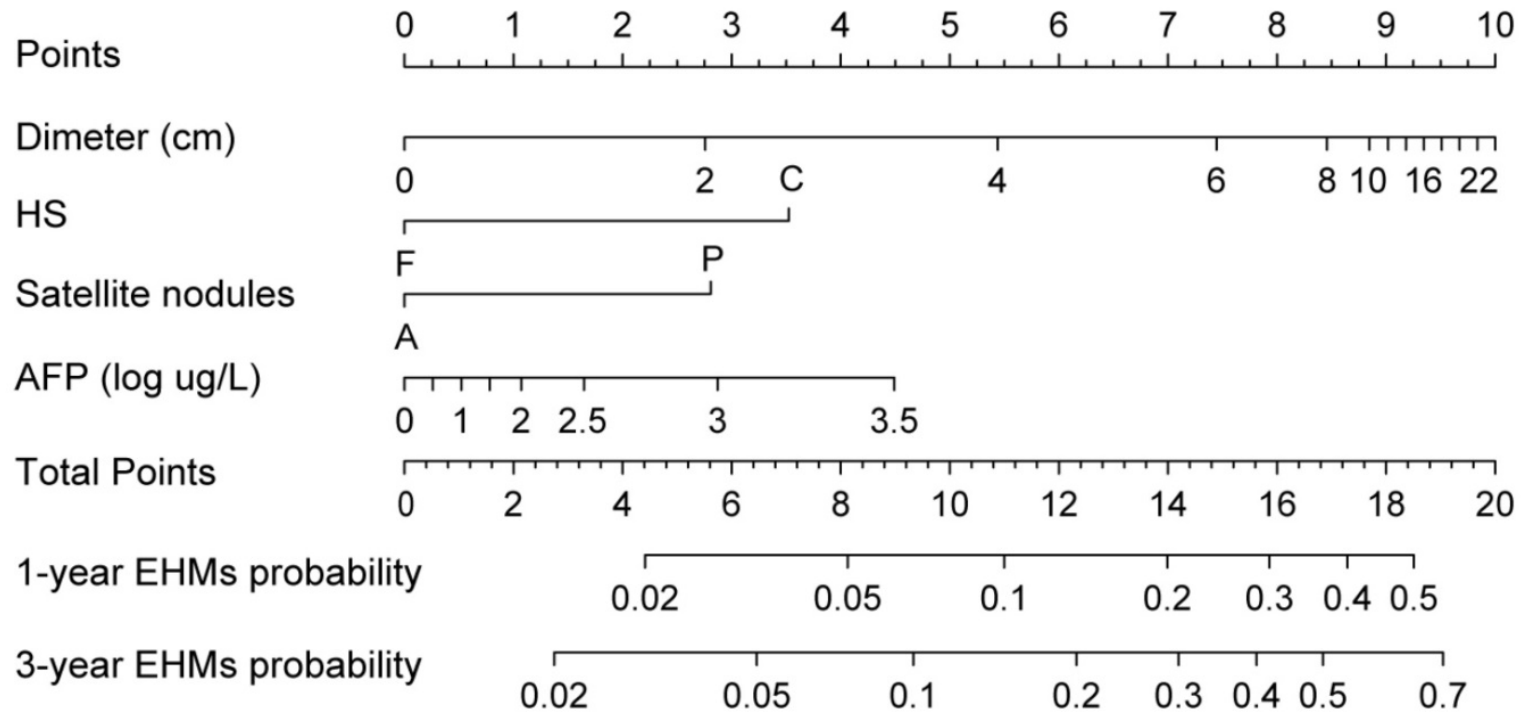

Figure 3. Nomogram predicting EHMs for HCC patients with adjuvant TACE following Hepatectomy. HS: histopathological structure of tumor; F: fine beam type; C: coarse beam type; A: absence; P: presence.

\section{Construction of the nomogram for EHMs}

A nomogram integrating these four significant independent variables to predict EHMs in the training cohort was then constructed (Figure 3). Patients who received TACE after hepatectomy had a higher score indicate a higher probability of developing EHMs. In order to calculate this score, the first step involved drawing a vertical line from the variable to the points scale in order to determine the corresponding points. This procedure was then repeated for each of the remaining risk factors. Finally, all values were summed on the total points axis, and a vertical line was drawn from the total points scale to the axis labeled "1-year EHMs probability" and "3-year EHMs probability" to calculate the probability of EHMs. 
(A) Training cohort

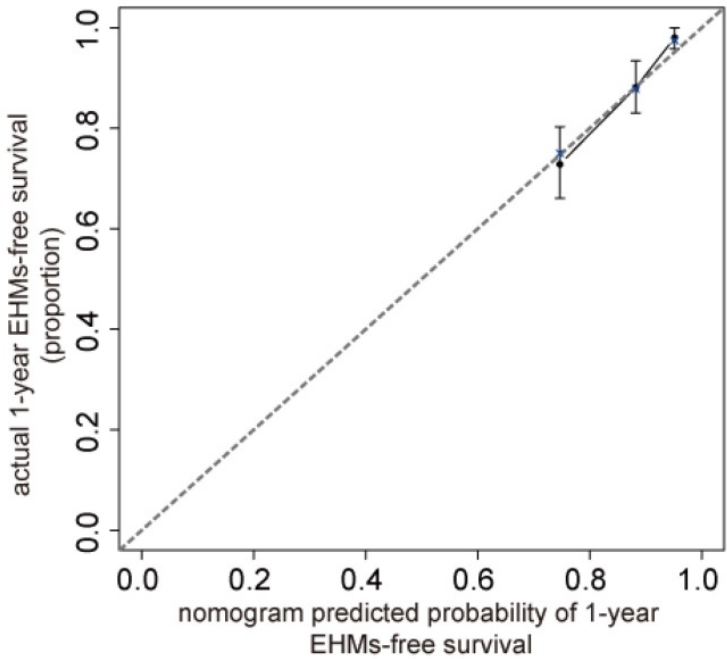

(B) Validation cohort

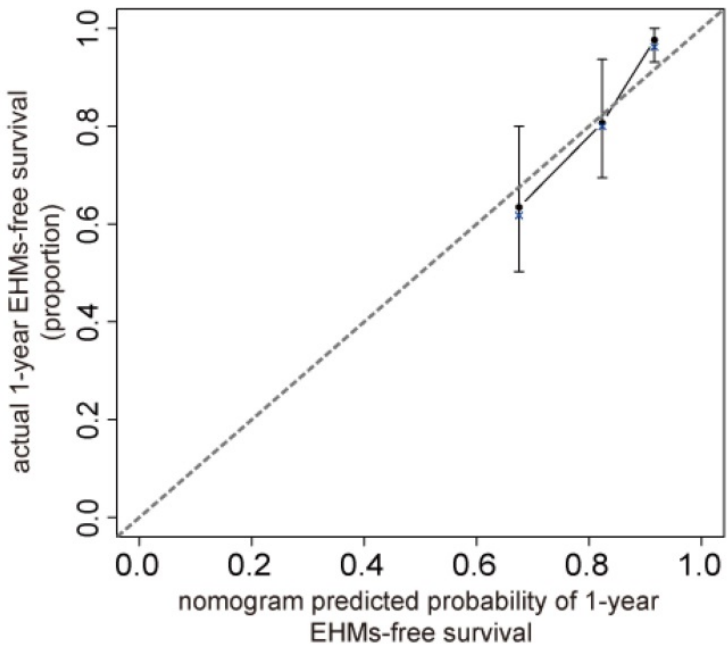

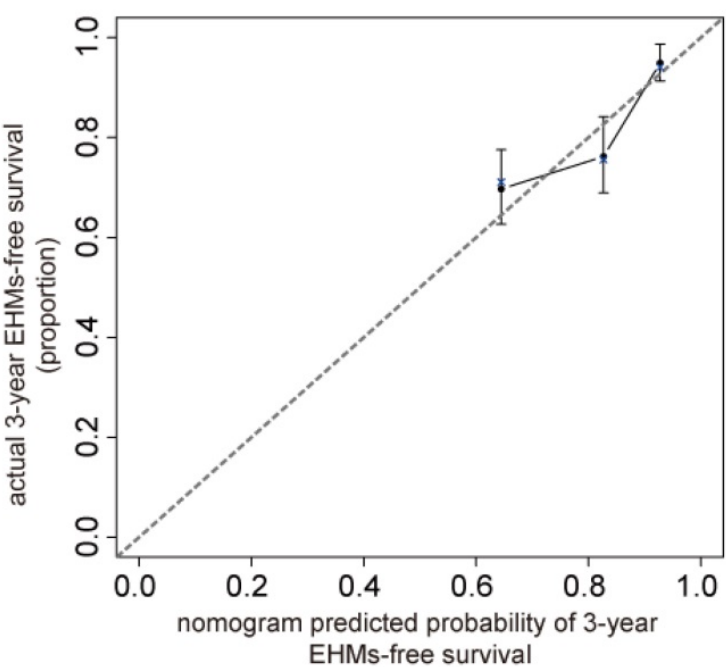

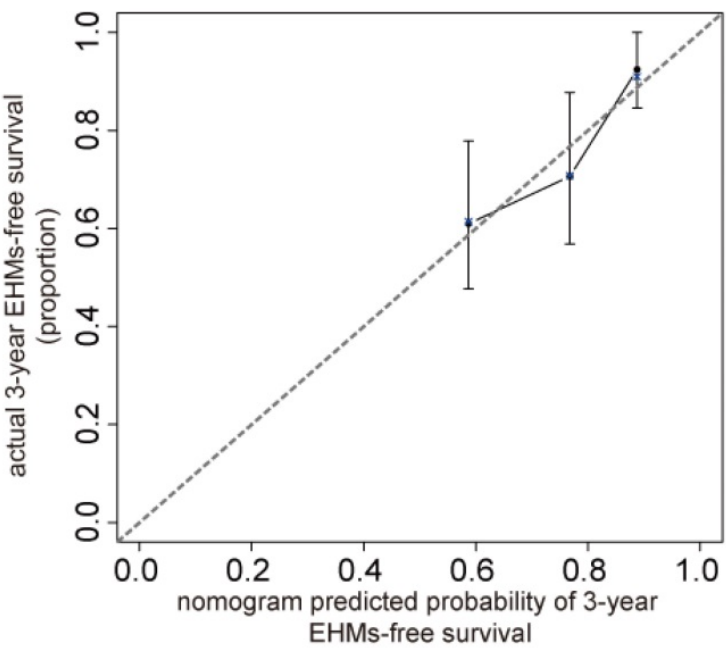

Figure 4. The calibration curve for nomogram at 1- and 3-year in the training cohort (A) and validation cohort (B). The nomogram predicted probability of EHMs-free survival is plotted on the $x$-axis; actual EHMs-free survival proportion is plotted on the $y$-axis. Thin gray dashed represents the reference line.

\section{Validation and performance of the prediction nomogram}

The C-index of our nomogram in the training cohort was 0.73 (95\% CI, 0.68- 0.78), and calibration plots showed good agreement between the estimated probability by nomogram and the actual proportion (Figure 4A). For the independent validation cohort, and the nomogram displayed a C-index of $0.71(95 \%$ CI, 0.63-0.79), which also represented a good calibration curve (Figure 4B). In addition, Figure 5 shows a plot of the time-dependent AUC, reflecting area under the ROC curve at all time points, indicating the comparative stability and adequate discriminative ability of our nomogram for predicting EHMs. It is clear that the AUC of the model is stable, which fluctuated around 0.75 both in the training and validation cohorts. Furthermore, the diagnostic capacity of the model is higher than that of any single one risk factor taken independently.

\section{Decision tree model for predicted probabilities of EHMs based on nomogram}

As noted previously, patients with higher scores had worse prognoses. Predicted probabilities of EHMs based on nomogram were divided into three groups using decision tree model by predicted risk scores. Patients who scored $>17$ were determined to be in the high risk group. Scores ranging $>12$ and $\leq 17$ resulted in classifying patients into the moderate risk group. Patients who scored $\leq 12$ were determined to be in the low risk group. We observed that $37.50 \%$ of patients in the high risk group had EHMs, while those in the moderate and low risk groups exhibited a rate of $20.11 \%$ and $5.66 \%$, respectively, after three years of follow-up in the training cohort. Similarly, patients in 
the high, moderate and low risk groups had EHMs with a rate of $38.46 \%, 21.43 \%$, and $15.91 \%$, respectively, after three years of follow-up in the validation cohort. Decision tree modeling was used to stratify probabilities of EHMs that were then used to plot Kaplan-Meier curves, both in the training and validation cohorts (all $p<0.05$ ) (Figure 6). It is clear that those patients who scored $\leq 12$ points had a much lower rate of EHMs.

\section{Discussion}

Adjuvant TACE is widely used in postoperative HCC patients with recurrence risk [23]. It was reported that patients who received adjuvant TACE

(A) Training cohort

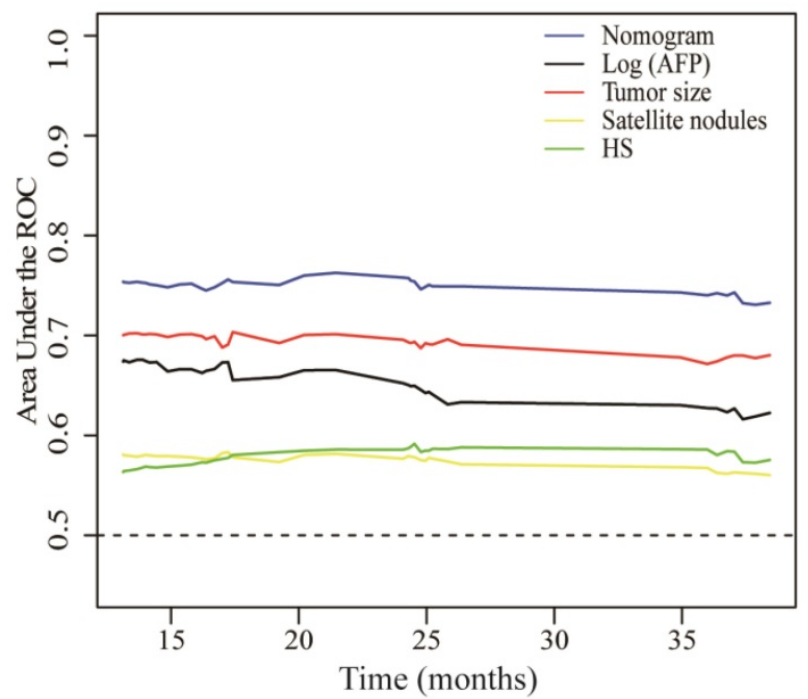

after hepatectomy had significantly higher survival [2, 3]. Frequently, HCC presents as advanced disease, and despite curative-intent surgical resection, EHMs were common in HCC patients who underwent TACE after hepatectomy [6, 24]. Precise information about prognosis is essential for decision-making and patient counseling. Although some progress has been made on the management of EHMs, no good method is available for predicting EHMs, thus limiting their early detection. At present, there are some tools known as nomograms to predict disease outcomes or assess risk based on specific characteristics of a patient in the clinical practice [25-27]. In this study, we developed a nomogram, which is a kind of statistical

\section{(B) Validation cohort}

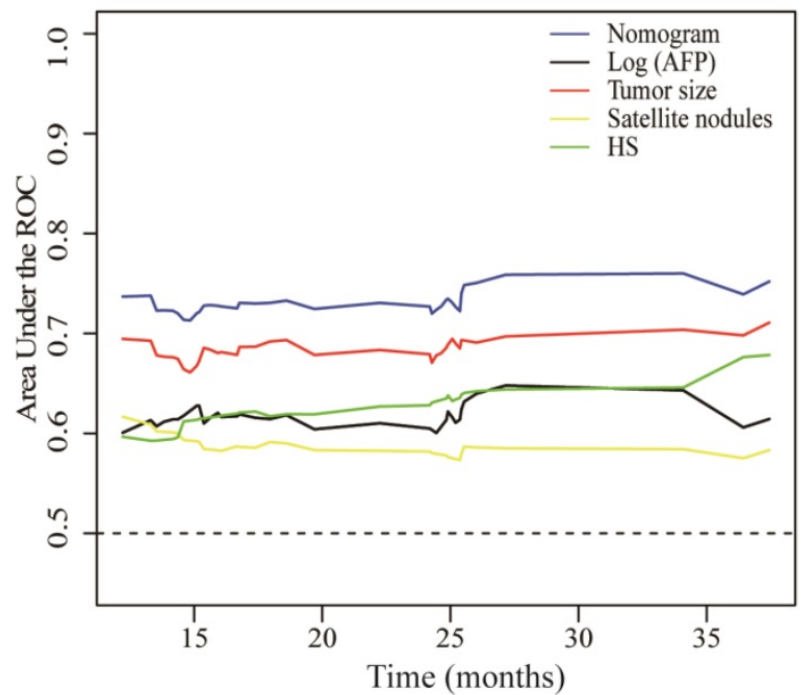

Figure 5. Time-dependent AUC plot for EHMs prediction model in the training cohort (A) and validation cohort (B). HS: histopathological structure of tumor.

(A) Training cohort

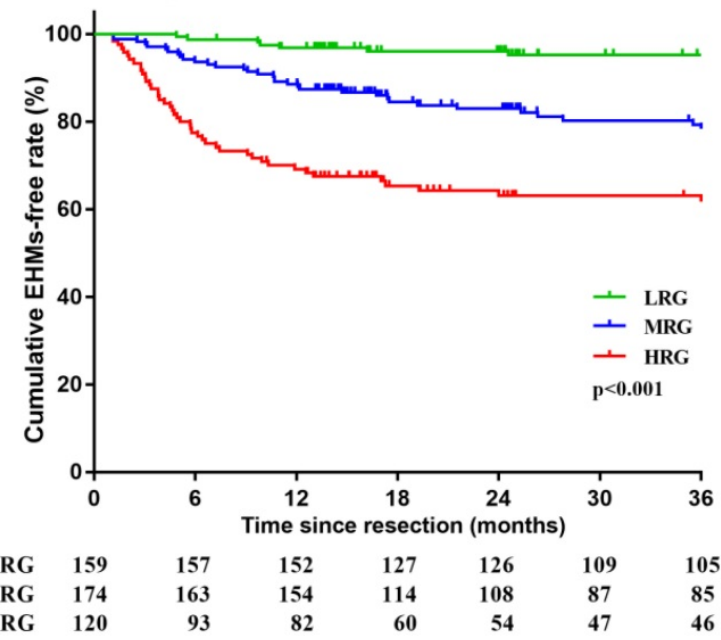

(B) Validation cohort

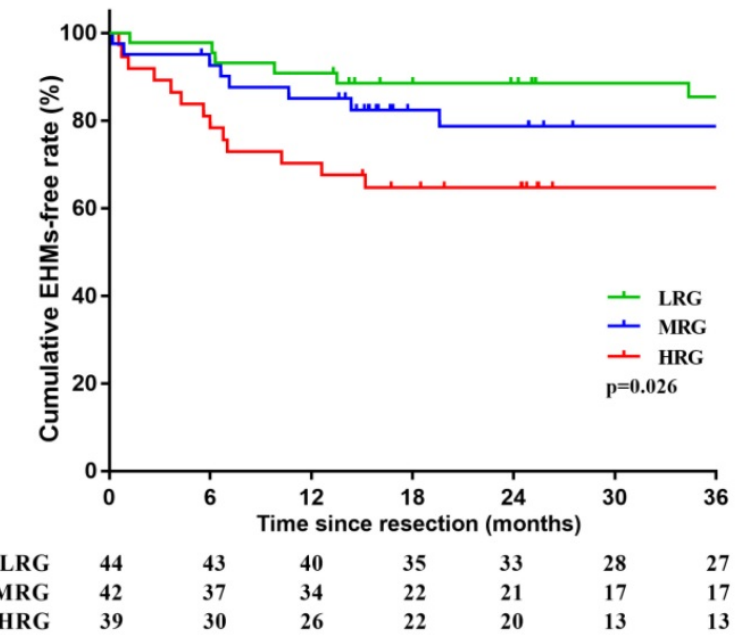

Figure 6. Kaplan-Meier survival analysis of HCC patients stratified into low-, moderate- and high-risk groups in the training cohort (A) and validation cohort (B). LRG: low risk group; MRG: moderate risk group; HRG: high risk group. 
tool able to predict the probability of EHMs for individual patients by assessing multiple risk factors. Based on patients in the training cohort who had undergone adjuvant TACE following hepatectomy for HCC, we created a statistically predictive nomogram of EHMs probability. To the best of our knowledge, this is the first clinical scoring system for EHMs prediction in HCC patients who had undergone adjuvant TACE following hepatectomy.

In the present research, we developed a nomogram that performed well with a C-index of 0.73 (95\% CI, 0.68 to 0.78 ) for prediction in the training cohort. Predictive performance was further certified by an external validation cohort with a C-index of 0.71 (95\% CI, 0.63 to 0.79 ). Furthermore, by using a decision tree model, patients were divided into three groups: high risk, > 17 points; moderate risk, $>12$ and $\leq 17$ points; and low risk, $\leq 12$ points. On the Kaplan-Meier curves, it is clear that those patients with low risk had a much lower rate of EHMs (Figure 6).

In this study, the nomogram included four variables consisting of presurgical baseline characteristics and pathological features of tumor. Our study suggests that large tumor diameter, coarse beam type of tumor histopathological structure, presence of satellite nodules and higher serum AFP level are significantly associated with an increased probability of EHMs. AFP is a routine tumor marker for HCC patients, higher AFP may suggest more advanced cancer burden and greater risk of remaining tumor and recurrence after surgery [28, 29]. In addition, many have reported that serum AFP is an important variable associated with HCC with EHMs $[30,31]$. Thus, AFP was previously included as an independent risk factor for establishing a nomogram to predict metastasis of HCC [32]. It was previously modeled as having linear effect on predictive results; However, the use of a linear model alone may not accurately reflect the effect of AFP on outcome. Therefore, it is essential to take the nonlinear effect of AFP into consideration. It is clear from the graph that the influence of AFP on the HR of EHMs was linear under a threshold of almost $2 \log (\mathrm{AFP}) \mathrm{ug} / \mathrm{L}$, after which it increased dramatically (Figure 2A).

As for tumor size which was also associated with a nonlinear effect on HR of EHMs. Studies have reported that tumor size is a vital predictive factor associated with HCC progression [33]. It also has been included in many staging systems for HCC. Overall survival among patients with a single-tumor $>5 \mathrm{~cm}$ was significantly lower than patients with a single-tumor $>2$ and $\leq 5 \mathrm{~cm}(\mathrm{p}<0.001)$ [34]. Moreover, huge HCC $(\geq 10 \mathrm{~cm})$ is an independent risk factor owing to a high risk for initial extrahepatic recurrence [35]. However, very few studies have reported on tumor diameter of HCC patients after partial hepatectomy and adjuvant TACE. The effect of tumor size on the risk of EHMs was linear up to approximately $6 \mathrm{~cm}$ diameter, after which the risk of EHMs increases more gradually (Figure 2B). The threshold effect of tumor size was also noted by Lim et al [36].

Other factors were also selected based on multivariate analyses, including the presence of satellite nodules and coarse beam type of tumor histopathological structure. Satellite nodules are an independent risk factor for tumor recurrence and patient survival [37]. The presence of satellite nodules was independently associated with the occurrence of postoperative complications [38]. In this study, tumor diameter and satellite nodules reflected the invasiveness of HCC, and they were significantly associated with EHMs. Patients who underwent TACE after curative resection with the presence of satellite nodules had a higher probability of EHMs (Figure 3). The tumor histopathological structure is mainly based on the number, arrangement and interstitial response of tumor cells, which are related to tumor differentiation, growth and dissemination. It includes fine beam type, coarse beam type, false gland tube type, compact type and hardened type. In pathological diagnosis, the histopathological structure of tumor exists interactively, so we distinguish it according to the main lesion types [39]. Fine beam type consists of 1-3 layers of cells, and the differentiation of tumor cells is better. Coarse beam type is formed by 20-30 cell layers, and the morphology of tumor cells is obviously abnormal. Also, the blood supply for coarse beam type is much more abundant; therefore, the invasiveness of this tumor type could be strengthened [40]. Thus, those patients classified as coarse beam type are at a high risk of EHMs.

In this study, we constructed a tool able to generate an individual's probability of developing EHMs by different prognostic and determinative variables. Using this model, the chance of developing EHMs based on data from individual cases could be calculated. Thus, the surgeon would be able to plan for case management early on to reduce both recurrence and EHMs after adjuvant TACE following HCC resection. The tool would also help the surgeon to determine which patients are good candidates for liver transplantation based on their risk for EHMs.

Our study does have some limitations. First, the data were acquired from a single institution, while it is generally necessary to validate such model against external centers with different geography. Second, most of our patients had a background of HBV 
infection and liver cirrhosis, making it necessary to validate this model against a different disease background. Third, we also do think that it is necessary to validate our model with larger sample size in the future. Besides, many studies have demonstrated the association between circulating tumor cells (CTCs)/cancer stem cell markers and HCC postoperative metastasis. Currently we mainly aimed to use the clinical routine available detections to predict EHMs. CTCs and cancer stem cell markers might be integrated into the nomogram in the future, if the assay could be more optimized and clinical applicable as well as enough data available. However, in spite of these shortcomings, our model may offer an alternative way to monitor the risk of EHMs in postoperative HCC patients.

In conclusion, we have constructed a reliable nomogram to predict the probability of EHMs in individual HCC patients who have undergone TACE after hepatectomy. This is an easy-to-use tool for the early diagnosis and prevention of EHMs and could benefit surgeons in decision-making.

\section{Acknowledgements}

This work was supported by the Science and Technology Commission of Shanghai Municipality [No.17JC1404500], National Natural Science Foundation of China [No. 81572072], Shanghai Medical Health Development Foundation [2016] 05 and [2018] 02.

\section{Ethical Approval}

All procedures performed in studies involving human participants were in accordance with the ethical standards of the institutional and/or national research committee and with the 1964 Helsinki declaration and its later amendments or comparable ethical standards. The study was approved by the Institutional Ethics Committee of Eastern Hepatobiliary Surgery Hospital, Second Military Medical University.

\section{Informed consent}

Informed consent was obtained from all individual participants included in the study.

\section{Competing Interests}

The authors have declared that no competing interest exists.

\section{References}

1. Forner A, Llovet JM, Bruix J. Hepatocellular carcinoma. Lancet. 2012; 379: $1245-1255$.

2. Zhong C, Guo RP, Li JQ, et al. A randomized controlled trial of hepatectomy with adjuvant transcatheter arterial chemoembolization versus hepatectomy alone for Stage III A hepatocellular carcinoma. J Cancer Res Clin Oncol. 2009; 135: 1437-1445.
3. Li Q, Wang J, Sun Y, et al. Efficacy of postoperative transarterial chemoembolization and portal vein chemotherapy for patients with hepatocellular carcinoma complicated by portal vein tumor thrombosis--a randomized study. World J Surg. 2006; 30: 2004-2011, 2012-2013.

4. Uka K, Aikata H, Takaki S, et al. Clinical features and prognosis of patients with extrahepatic metastases from hepatocellular carcinoma. World J Gastroenterol. 2007; 13: 414-420.

5. Yang Y, Nagano H, Ota H, et al. Patterns and clinicopathologic features of extrahepatic recurrence of hepatocellular carcinoma after curative resection. Surgery. 2007; 141: 196-202.

6. Ollila DW. Complete metastasectomy in patients with stage IV metastatic melanoma. Lancet Oncol. 2006; 7: 919-924.

7. Kawamura M, Nakajima J, Matsuguma H, et al. Surgical outcomes for pulmonary metastases from hepatocellular carcinoma. Eur J Cardiothorac Surg. 2008; 34: 196-199.

8. Zhou LY, Zeng ZC, Fan J, et al. Radiotherapy treatment of adrenal gland metastases from hepatocellular carcinoma: clinical features and prognostic factors. Bmc Cancer. 2014; 14: 878 .

9. Zhong JH, Li LQ. Postoperative adjuvant transarterial chemoembolization for participants with hepatocellular carcinoma: A meta-analysis. Hepatol Res. 2010; 40: 943-953.

10. Palmer DH, Midgley RS, Mirza N, et al. A phase II study of adoptive immunotherapy using dendritic cells pulsed with tumor lysate in patients with hepatocellular carcinoma. Hepatology. 2009; 49: 124-132.

11. Huang G, Lau WY, Wang ZG, et al. Antiviral therapy improves postoperative survival in patients with hepatocellular carcinoma: a randomized controlled trial. Ann Surg. 2015; 261: 56-66.

12. Chan $\mathrm{KM}, \mathrm{Yu} \mathrm{MC}, \mathrm{Wu} \mathrm{TJ}$, et al. Efficacy of surgical resection in management of isolated extrahepatic metastases of hepatocellular carcinoma. World J Gastroenterol. 2009; 15: 5481-5488.

13. Zeng ZC, Tang ZY, Fan J, et al. Consideration of role of radiotherapy for lymph node metastases in patients with HCC: retrospective analysis for prognostic factors from 125 patients. Int J Radiat Oncol Biol Phys. 2005; 63: 1067-1076.

14. Zhou LY, Zeng ZC, Fan J, et al. Radiotherapy treatment of adrenal gland metastases from hepatocellular carcinoma: clinical features and prognostic factors. Bmc Cancer. 2014; 14: 878.

15. He J, Zeng ZC, Tang ZY, et al. Clinical features and prognostic factors in patients with bone metastases from hepatocellular carcinoma receiving external beam radiotherapy. Cancer-Am Cancer Soc. 2009; 115: 2710-2720.

16. Llovet JM, Ricci S, Mazzaferro V, et al. Sorafenib in advanced hepatocellular carcinoma. N Engl J Med. 2008; 359: 378-390.

17. Kudo M, Finn RS, Qin S, et al. Lenvatinib versus sorafenib in first-line treatment of patients with unresectable hepatocellular carcinoma: a randomised phase 3 non-inferiority trial. Lancet. 2018; 391: 1163-1173.

18. Qin S, Bai Y, Lim HY, et al. Randomized, multicenter, open-label study of oxaliplatin plus fluorouracil/leucovorin versus doxorubicin as palliative chemotherapy in patients with advanced hepatocellular carcinoma from Asia. J Clin Oncol. 2013; 31: 3501-3508.

19. Qiu SJ, Zhou ZG, Shen F, et al. A multicenter, randomized, observation-controlled clinical trial to evaluate the efficacy and safety of thymalfasin adjuvant therapy in patients with HBV-related HCC after curative resection - first announcement of the protocol. Expert Opin Biol Ther. 2015; 15 Suppl 1: S133-S137.

20. Song $\mathrm{X}, \mathrm{Li} \mathrm{Y}$, Zhang $\mathrm{H}$, et al. The anticancer effect of Huaier (Review). Oncol Rep. 2015; 34: 12-21.

21. Balachandran VP, Gonen M, Smith JJ, et al. Nomograms in oncology: more than meets the eye. Lancet Oncol. 2015; 16: e173-e180.

22. EASL-EORTC clinical practice guidelines: management of hepatocellular carcinoma. J Hepatol. 2012; 56: 908-943.

23. Gao Q, Qiu SJ, Fan J, et al. Intratumoral balance of regulatory and cytotoxic T cells is associated with prognosis of hepatocellular carcinoma after resection. J Clin Oncol. 2007; 25: 2586-2593.

24. Zhou YM, Zhang XF, Yu F, et al. Efficacy of surgical resection for pulmonary metastases from hepatocellular carcinoma. Med Sci Monit. 2014; 20: 1544-1549.

25. Wang Y, Li J, Xia Y, et al. Prognostic nomogram for intrahepatic cholangiocarcinoma after partial hepatectomy. J Clin Oncol. 2013; 31: 1188-1195.

26. Hyder $\mathrm{O}$, Marques $\mathrm{H}$, Pulitano $\mathrm{C}$, et al. A nomogram to predict long-term survival after resection for intrahepatic cholangiocarcinoma: an Eastern and Western experience. Jama Surg. 2014; 149: 432-438.

27. Lei $\mathrm{Z}, \mathrm{Li} \mathrm{J}, \mathrm{Wu} \mathrm{D}$, et al Nomogram for Preoperative Estimation of Microvascular Invasion Risk in Hepatitis B Virus-Related Hepatocellular Carcinoma Within the Milan Criteria. Jama Surg. 2016; 151: 356-363.

28. Ren ZG, Lin ZY, Xia JL, et al. Postoperative adjuvant arterial chemoembolization improves survival of hepatocellular carcinoma patients with risk factors for residual tumor: a retrospective control study. World J Gastroenterol. 2004; 10: 2791-2794.

29. Shen JY, Li C, Wen TF, et al. Alpha fetoprotein changes predict hepatocellular carcinoma survival beyond the Milan criteria after hepatectomy. J Surg Res. 2017; 209: 102-111.

30. Uchino K, Tateishi R, Shiina S, et al. Hepatocellular carcinoma with extrahepatic metastasis: clinical features and prognostic factors. Cancer-Am Cancer Soc. 2011; 117: 4475-4483. 
31. Senthilnathan S, Memon K, Lewandowski RJ, et al. Extrahepatic metastases occur in a minority of hepatocellular carcinoma patients treated with locoregional therapies: analyzing patterns of progression in 285 patients. Hepatology. 2012; 55: 1432-1442.

32. Li J, Liu Y, Yan Z, et al. A nomogram predicting pulmonary metastasis of hepatocellular carcinoma following partial hepatectomy. Br J Cancer. 2014; 110: $1110-1117$.

33. Forner A, Reig ME, de Lope CR, et al. Current strategy for staging and treatment: the BCLC update and future prospects. Semin Liver Dis. 2010; 30: 61-74.

34. El-Fattah MA, Aboelmagd M, Elhamouly M. Prognostic factors of hepatocellular carcinoma survival after radiofrequency ablation: A US population-based study. United European Gastroenterol J. 2017; 5: 227-235.

35. Wakayama K, Kamiyama T, Yokoo H, et al. Huge hepatocellular carcinoma greater than $10 \mathrm{~cm}$ in diameter worsens prognosis by causing distant recurrence after curative resection. J Surg Oncol. 2017; 115: 324-329.

36. Lim C, Mise $Y$, Sakamoto $Y$, et al. Above $5 \mathrm{~cm}$, size does not matter anymore in patients with hepatocellular carcinoma. World J Surg. 2014; 38: 2910-2918.

37. Hwang S, Lee YJ, Kim KH, et al. Long-Term Outcome After Resection of Huge Hepatocellular Carcinoma $\geq 10 \mathrm{~cm}$ : Single-Institution Experience with 471 Patients. World J Surg. 2015; 39: 2519-2528.

38. Doussot A, Lim C, Lahat E, et al. Complications after Hepatectomy for Hepatocellular Carcinoma Independently Shorten Survival: A Western, Single-Center Audit. Ann Surg Oncol. 2017.

39. Rui J. Modern Diagnostics and Treatment of Hpatocellular Carcinoma. 1 ed: Tsinghua University Press, 2004.

40. Becker FF. Morphological classification of mouse liver tumors based on biological characteristics. Cancer Res. 1982; 42: 3918-3923. 